\title{
Dynamique des apports en nutrients azotés et phosphorés à la rivière Aveyron
}

\author{
E. Chauvet 1 \\ M. Prat ${ }^{2}$ \\ J.N. Tourenq ${ }^{3}$
}

A partir de données physico-chimiques acquises sur le cours supérieur de l'Aveyron (octobre 79 à septembre 80 ), la nature des apports en nutrients azotés et phosphorés, Ieur localisation et le rôle auto-épurateur de certains tronçons sont mis en évidence.

\section{Dynamics of contributions of nitrogen and phosphorus to the river Aveyron.}

From the start of the acquisition of physical-chemical data from the upper reaches of the Aveyron (October 1979 - September 1980), the nature of contributions of nitrogen and phosphorus nutrients, their location and the self-purifying role of certain sections are described.

Le cours supérieur de l'Aveyron (amont de Rodez) présente des signes manifestes d'eutrophisation. $\mathrm{Si}$, même dans les zones lotiques, le phytoplancton est peu concerné par ce phénomène, le périphyton (essentiellement des Diatomées benthiques) et les macrophytes aquatiques traduisent, par leur important développement, une évolution en liaison avec l'apport de nutrients (Chauvet 1981).

Nous nous sommes efforcés dans ce travail de localiser et définir la nature des apports en nutrients le long du Haut-Aveyron et de ses affluents au cours d'un cycle annuel.

\section{1. - Rivière étudiée}

L'Aveyron est un affluent rive droite du Tarn. La région étudiée correspond au bassin supérieur de son cours, en amont de Rodez. Quinze stations de

1. Centre d'Ecologie des Ressources Renouvelables (C.N.R.S.), 29. rue J. Marvig, 31055 Toulouse Cedex.

2. Institut de Mécanique des Fluides (L.A.). 2 ne Camichel, 31071 Toulouse Cedex.

3. Laboratoire d'Hydrobiologie, E.R.A. 702 du C.N.R.S., Université Paul Sabatier, 118, route de Narbonne, 31062 Toulouse Cedex. mesure ont été choisies sur le cours principal et les affluents (figure 1).

Les affluents de la rive droite (le Merdans, la Serre) présentent une résistivité faible, un $\mathrm{pH}$ élevé, une alcalinité et une dureté calcique fortes et peu de silice. Ils s'opposent aux affluents de la rive gau. che (la Verlenque, l'Olip, le Galtiès, la Loubière et la Briane) dont la résistivité est élevée, le pH plus faible, l'alcalinité et la dureté calcique faibles et la teneur en silice importante; une teneur en silice et une résistivité beaucoup plus élevées caractérisent les stations O1, O2, G et surtout L. Ces différences sont dues à la nature géologique des sous-bassins versants drainés par ces affluents : globalement, le sud (affluents rive gauche) est un massif cristallin, alors que le nord (affluents rive droite) est constitué de causses calcaires.

\section{2. - Méthodes}

Les méthodes de dosage d'azote et de phosphore sont celles utilisées pour mettre en évidence dans les eaux, de faibles quantités de nutrients.

L'ammoniaque a été dosé selon la méthode au bleu-indophénol (de Dora Sheiner) avec une préci- 
sion de $2 \mu \mathrm{g} . \mathrm{l}^{1} \mathrm{l}$ d'azote ammoniacal ; les nitrites, dosés selon la méthode de Griess, ont été décelés avec une précision de 0,2 à $2 \mu \mathrm{g} . \mathrm{l}^{-1} \mathrm{~d}$ 'azote nitreux ; les nitrates ont été dosés selon la méthode au salicylate de sodium avec une précision de 2 à $10 \mu \mathrm{g} \cdot \mathrm{l}^{-1}$ d'azote nitrique. Les orthophosphates et le phosphore total dissous (après minéralisation en milieu acide) ont été dosés selon la méthode de Stephens, avec une précision allant de 0,1 à $2 \mu \mathrm{g} . \mathrm{l}^{-1}$ de phosphore.

Ces dosages d'azote et de phosphore, et les mesures de débit instantané correspondantes ont été effectuées mensuellement au niveau de chaque station (tableau I).

Tableau I. Mesures physico-chimiques réalisées aux 15 stations d'octobre 1979 à novembre 1980.

1: concentration en ammoniaque $\left(\mu \mathrm{g} \cdot \mathrm{l}^{-1} \mathrm{~N}-\mathrm{NH}_{4}\right)$;

2 : concentration en nitrites $\left(\mu \mathrm{g} . \mathrm{l}^{-\mathrm{i}} \mathrm{N}-\mathrm{NO}_{2}\right)$;

3: concentration en nitrates $\left(\mu \mathrm{g} \cdot \mathrm{l}^{-1} \mathrm{~N}-\mathrm{NO}_{3}\right)$;

4 : concentration en orthophosphates $\left(\mu \mathrm{g} \cdot \mathrm{l}^{-1} \mathrm{P}-\mathrm{PO}_{4}\right)$;

5: concentration en phosphore total dissous $\left(\mu \mathrm{g} . \mathrm{l}^{-1} \mathrm{P}\right)$;

6: débit instantané (1.5 $\left.{ }^{-1}\right)$.

\begin{tabular}{|c|c|c|c|c|c|c|c|c|c|c|c|c|}
\hline & $\alpha \leq 1$ & tor. & Des. & Jen. & $\mathrm{F}_{44}$ & Marz & Avrit| & Mol & fuin & $34+1$, & Howt & Fent. \\
\hline न1 & - & f & (1) & B & 135 & 12 & 5 & 14 & $\bar{B}$ & 8 & 38 & $7 \%$ \\
\hline$v$ & - & g & 1 & 99 & 395 & a & E & 5 & 1 & z & 31 & $x$ \\
\hline $\mathrm{M}$ & $*$ & 3 & 36 & 358 & $m$ & a & * & . & . & * & . & - \\
\hline $\mathrm{Hz}$ & m & 214 & ind & 9 & 436 & $16 \mathrm{t}$ & $g$ & $m$ & 154 & 87 & 5II & 249 \\
\hline 01 & . & $2 \theta$ & 17 & 18 & 63 & 28 & $\theta$ & B & 5 & 126 & 418 & 38 \\
\hline 02 & " & 42 & 75 & Ias & $E 7$ & 32 & 22 & 47 & 22 & 29 & 55 & 94 \\
\hline ค3 & $n$ & 34 & B & 17 & so & 15 & 32 & 5 & 18 & 48 & 03 & $y$ \\
\hline $\mathbf{m}$ & $\pi$ & 36 & 35 & !64 & 24 & is & 1 & 7 & 98 & 29 & 36. & 72 \\
\hline s & m & b? & 2 & 38 & 12 & 2 & Ia & $a$ & 7 & 58 & II & 16 \\
\hline As & HE & 19 & 35 & $n$ & 41 & LBP & $\$$ & 129 & 98 & 195 & 6 & 81 \\
\hline 6 & - & 78 & s & $\mathrm{s}$ & 14 & a & 13 & 11 & 18 & 10 & 18 & 8 \\
\hline L & m & g & 1 & 1 & 5 & 2 & 9 & 13 & 1 & $g$ & a & a \\
\hline As & - & 8 & 16 & 24 & a & 53 & 31 & 28 & 3 & $3 e$ & $\pi$ & $\mathrm{S}_{4}$ \\
\hline B & . & 7 & 6 & 94 & 1 & 78 & 7 & E & 73 & 51 & 161 & 12 \\
\hline m? & ael. & 37 & b5 & 269 & 174 & 2a & 486 & 458 & 571 & 579 & 528 & 169 \\
\hline
\end{tabular}

3

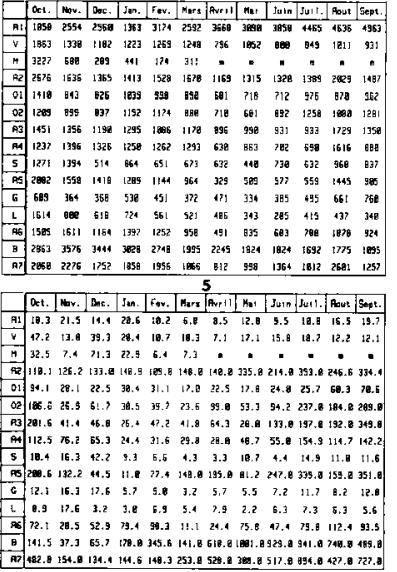

\begin{tabular}{|c|c|c|c|c|c|c|c|c|c|c|c|}
\hline \\
\hline$a x+$. & $10 \%$ & Dace. & Jon. & for. & Mars: & Afr, & $\begin{array}{ll}1 \\
\text { nal }\end{array}$ & Puin & Tsuit. & Inour & $15 x=$ \\
\hline .5 & .7 & 2.1 & .3 & 1.1 & 8.9 & 1.4 & 1.7 & .1 & 2.9 & 1.3 & 2.5 \\
\hline 2.9 & is & 2.5 & 8.8 & .7 & 0,8 & 0.1 & 1.5 & s & .6 & Q.8 & a . \\
\hline 18.5 & 1.7 & 18.9 & 6.2 & 2.4 & 1.5 & - & " & - & - & • & . \\
\hline 17.2 & 9.z & 13.2 & the & 1.2 & 206.8 & 86.6 & 12.1 & 46.8 & 20.5 & 62.4 & 461.2 \\
\hline 7.8 & 3.8 & 8. 4 & 2.5 & 2.2 & 11.7 & 5.8 & E.8 & 1.8 & 12.8 & 2.6 & $6 \quad 2.1$ \\
\hline 7.8 & 3.8 & 18.5 & 3.1 & 1.2 & 18.3 & 12.1 & 2.8 & 9.8 & 21.7 & 12.1 & 155.4 \\
\hline 19.8 & 5.5 & 9.2 & 3.9 & 5.5 & 18.1 & $1: 3.3$ & 111.6 & 16.6 & 20.1 & 35.6 & $\begin{array}{l}84.3 \\
\end{array}$ \\
\hline 9.8 & 4.5 & 14.7 & 5.4 & 2.7 & 8.6 & 7,8 & 8.6 & 9.5 & 15.7 & 22.6 & $\begin{array}{l}65.3 \\
6.3\end{array}$ \\
\hline 3.8 & 8 & 0.8 & .9 & 1.? & .9 & 2.6 & 3.2 & 3.5 & 2.3 & 3.4 & $4 \quad 1.7$ \\
\hline 23.4 & 4.5 & 18.8 & 2.5 & 2.4 & 8.8 & 42.1 & 18.3 & 18.4 & 21.8 & 21.1 & $1 \quad 13.7$ \\
\hline 0.8 & 3.8 & 2.6 & 1.5 & 1.3 & .3 & 0.8 & 1.2 & . & . & a. a & .2 \\
\hline 8.8 & 1.8 & A. 8 & O.8 & .8 & 8.8 & 8.8 & B.B & 8.8 & -1 & 8. 8 & .1 \\
\hline 18.6 & 4.9 & 17.8 & 3.4 & 4.6 & 5.4 & 8.4 & 02.1 & 18.1 & 15.1 & 77.8 & $8 \quad 1.9$ \\
\hline 21.2 & $15, \mathrm{e}$ & 14.8 & 19.4 & 16.1 & 1113.1 & 29.7 & 23.9 & 79.9 & 28.9 & 181.5 & 32.1 \\
\hline 155.8 & 10.8 & 62.8 & 29.5 & 102.5 & & 02.4 & s.9. & & 2137,6 & & $5: 81,2$ \\
\hline \multicolumn{12}{|c|}{4} \\
\hline$\theta=1$. & I 3 tov. & sace. & $3 \mathrm{~m}$. & Ifor. & $\mid \begin{array}{l}\text { naras } \\
\end{array}$ & Avrril| & 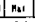 & Soin & Ts=1. & Trout & $t$ Sent \\
\hline 1118 & 14.8 & 15.8 & 18.6 & 18.-2 & 4.9 & 3.6 & 87.5 & 22.2 & 5.5 & 31.5 & 518.8 \\
\hline 27.8 & 13.5 & 12.7 & 18.5 & 7.5 & 8.7 & 4.4 & 1.2 & 13.4 & 411.2 & 9.8 & a 1.4 \\
\hline 27.6 & 1.8 & 39.5 & 7.8 & 1.1 &.$t$ & - & 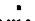 & - & - & & \\
\hline 48.3 & 94.5 & 91.1 & 85.4 & 81.4 & 38.2 & & & & & 8256.8 & 8311.8 \\
\hline 61.3 & 25.8 & 18.8 & 31.3 & 28.4 & 14.2 & 7.8 & 18.5 & $5 \quad 37.8$ & 2.3 & 47.5 & 546.8 \\
\hline 4.4 & 35.1 & 32,8 & 28.4. & 35.1 & 25.2 & 78.8 & 51.5 & 63.2 & 211.8 & Q 1110. & 227,4 \\
\hline 2.6 & 35.6 & 36.8 & 30.2 & $\gamma 0,1$ & 34.8 & 57.9 & 33,2 & 185.8 & 8 & $815+.7$ & 7315 \\
\hline 137,4 & 27. & 48.2 & 15.1 & 35. & 27.6 & 12.6 & 31.8 & 51.1 & 1 \&55. 3 & 3 \$8. 8 & 8102.8 \\
\hline 1.4 & i:? & 18.8 & 18.6 & 2.8 & 1.3 & .6 & .7 & 1.4 & 2.1 & 1.8 & 1.8 \\
\hline 221.5 & 81.8 & 71.9 & 68.9 & 84. & 97.6 & 78.9 & 41,5 & 159,7 & 246.8 & 8124.8 & 0371.8 \\
\hline 2,8 & 1.1 & .5 & 2.8 & 2.3 & .9 & .1 & 1.2 & 2.3 & 1.6 & 1.1 & 4.3 \\
\hline 2,3 & 7.9 & .2 & 2.2 & .3 & 0.8 & .2 & 1.9 & .7 & 1.1 & 4 & 2.5 \\
\hline \begin{tabular}{|l}
85.1 \\
617.9
\end{tabular} & $\begin{array}{l}78.5 \\
23.6\end{array}$ & $\begin{array}{l}31.6 \\
55.6\end{array}$ & & & & & & & & & \\
\hline s. & 55.6 & 166.8 & 189.8 & 128.9 & & Q 318.6. & 239.8 & 8 ४ 481.8 & 875.0 & 0365.4 &, 577 \\
\hline \multicolumn{12}{|c|}{6} \\
\hline Set. & now. & int. & $\mathrm{Im}$. & Fen. & kars & anr 1] & 20.1 & Juin & [Juil.] & Aout & Sefti \\
\hline 138 & $m$ & 85 & 05 & 131 & 2 & 41 & 73 & $\overline{32}$ & ? & 15 & \\
\hline 64: & $5: 4$ & 354 & 39. & 648 & 978 & 189 & 337 & 109 & "1 & 74 & 39 \\
\hline 153 & 328 & 76 & 258 & sil & 23! & a & B & . & a & , & \\
\hline 26 & 1,158 & 806 & 928 & 15318 & 998 & 388 & sse & 3 & 198 & $26 \mathrm{~d}$ & i68 \\
\hline 11868 & 958 & 73e & 738 & 1:ARE & 978 & 378 & 158 & 223 & B?" & 170 & $2 n$ \\
\hline 1188 & 258 & zase & 7 & I18 & 678 & $3 n$ & 58 & 25 & 329 & 138 & \\
\hline 3788 & $2 ! 8$ & $16: 6$ & 1650 & $27 \mathrm{~B}$ & 1058 & 38 & 168 & 525 & 510 & 390 & \\
\hline 25 & 5358 & 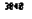 & $35 \%$ & 4840 & $518 \mathrm{E}$ & $i<28$ & 1989 & 10 & 745 & 1060 & \\
\hline 30 & 1958 & 2030 & 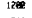 & 1378 & 1078 & 515 & 666 & 439 & (4) & 361 & 261 \\
\hline 208 & 7318 & 5870 & 4798 & 6218 & 6578 & (3) & $26 \div 5$ & 1585 & 1945 & 1421 & 816 \\
\hline I & 3 & 29 & 30 & 29 & 158 & 4 & ${ }^{24}$ & 23 & 2) & 5 & \\
\hline 3 & 268 & 220 & 178 & 158 & 128 & 15 & & & & & \\
\hline ट्टा & & & 6978 & тरвн & & 30 & $165 \mathrm{e}$ & 7420 & 1638 & เ69 & \\
\hline & 673 & 525 & 432 & 5 & 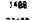 & 218 & 150 & 198 & & 25 & \\
\hline & & & 340 & & & & & & t698 & 165 & \\
\hline
\end{tabular}




\section{3. - Résultats et discussion}

a) Etude comparative des concentrations.

En A2, A5 et surtout A7, on observe de fortes concentrations en $\mathrm{NH}_{4}, \mathrm{NO}_{2}$ et ortho- $\mathrm{PO}_{4}$ (plus de 100 $\mu \mathrm{g} . \mathrm{I}^{-1} \mathrm{P}$ ). En A3, A4 et A6, on remarque une très nette diminution des teneurs par rapport aux stations précédentes, en particulier pour $\mathrm{NO}_{2}$, ortho$\mathrm{PO}_{4}$ et $\mathrm{P}$ total dissous. Les concentrations sont sen- siblement plus élevées en $0_{2}$ qu'en $O_{1}$. Les teneurs en azote et phosphore de la Serre (S), du Galtiès (G) et de la Loubière (L) sont très faibles. La Briane (B) présente de fortes concentrations en $\mathrm{NH}_{4}, \mathrm{NO}_{2}$. $\mathrm{NO}_{3}$ ainsi que les plus fortes teneurs en phosphore du bassin.

A l'issue de cette analyse, on peut tracer une carte indiquant l'importance relative des teneurs en azote et en phosphore aux différentes stations d'étude (fig. I).

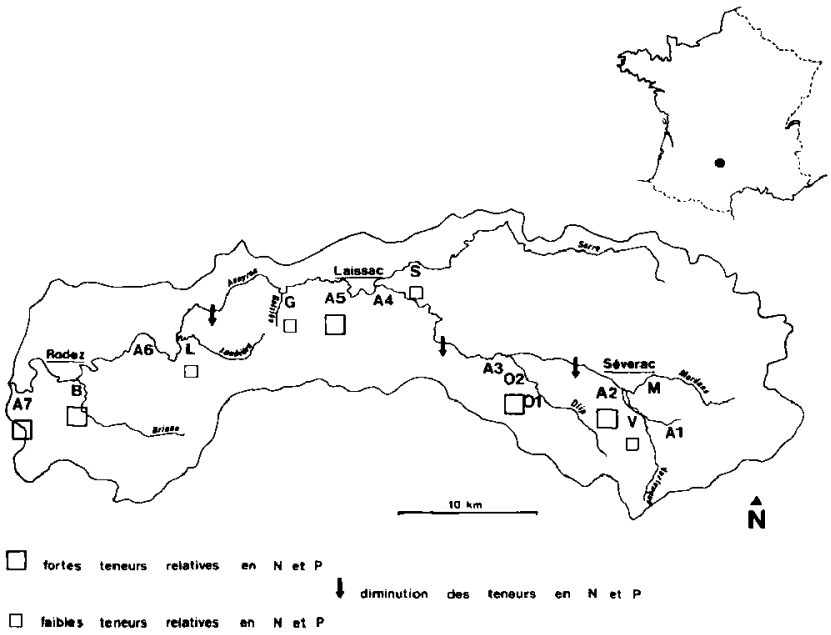

Fig. 1. Carte du haut-bassin de l'Aveyron indiquant l'importance relative des teneurs en azote et en phosphore aux différentes stations étudiées.

A1 I'Aveyron à sa source ; V la Verlanque; $M$ le Merdans ; A2 l'Aveyron à l'aval de Séverac; o1 l'Olip à Recoules; 02 l'olip à Varès; A3 l'Aveyron à Bézonnes ; A4 l'Aveyron à Manson; $\mathrm{S}$ la Serre à Coussergues; $\mathbf{A} 5$ l'Aveyron à Laissac; $\mathrm{G}$ le Galtiès ; $\mathrm{L}$ la Loubière ; A6 l'Aveyron à Roquette : B la Briane au Monastère ; A7 l'Aveyron en aval de Rodez.

b) Etude des relations concentrations-débits.

D'après Manczak et Florczyk (1971) et Hall (1970), on peut résumer à trois cas simples les différents types de pollution d'une rivière (Belamie, 1978):

- cours d'eau principalement pollué par les rejets ponctuels et constants proches de la station de mesure; la fonction $C=f(Q)$ (avec, $C$ : concentration et $Q$ : débit) est décroissante et de type hyperbolique :

- cours d'eau où le lessivage est prépondérant ; la fonction $C=f(Q)$ est croissante et de divers types : linéaire, exponentielle, logarithmique ; 
- cours d'eau moyennement pollué ; la fonction $C$ $=\mathbf{f}(\mathbf{Q})$ est composite. L'apport en éléments fertilisants occasionné par les rejets (principal facteur de pollution pour les faibles débits) tend à être mas. qué par les apports dus au lessivage lorsque les débits augmentent.

Les figures 2, 3, 4 et 5 donnent une représentation graphique des relations concentration-débit pour $\mathrm{NH}_{4}, \mathrm{NO}_{3}$, ortho- $\mathrm{PO}_{4}$ et $\mathbf{P}$ total dissous (les concentrations en ordonnée et les débits en abscisse sont exprimées en valeur relative).
Rejets ponctuels :

L'examen des représentations graphiques de ces relations pour $\mathrm{NH}_{4}$, ortho- $\mathrm{PO}_{4}$ et $\mathrm{P}$ total dissous montre que :

- les stations $\mathrm{A} 2, \mathrm{O} 2, \mathrm{~A} 3$ et $\mathrm{A} 7$ sont de façon très nette sous l'influence de rejets ponctuels. Ceci est particulièrement évident à la station $\mathrm{A} 7$ pour $\mathrm{NH}_{4}$ et aux stations $\mathrm{A} 2$ et $\mathrm{O} 2$ pour ortho- $\mathrm{PO}_{4}$ et $\mathrm{P}$ total dissous, par exemple ;
Fig. 2. Relations concentration-débit pour l'ammoniaque $(\mathrm{O}$ : valeur manquante ; concentrations en ordonnée et débits en abscisse sont exprimés en valeur relative du maximum pour chaque station).

Fig. 3. Relations concentration-débit pour les nitrates (concentrations en ordonnée et débits en abscisse sont exprimés en valeur relative).
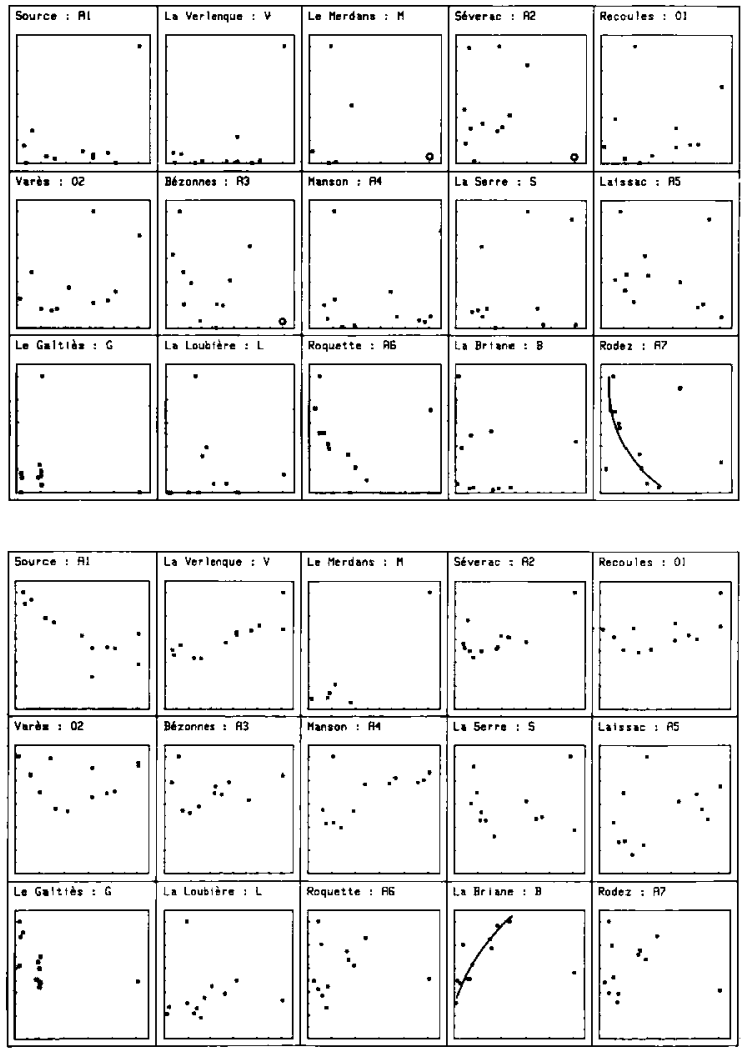
Fig. 4. Relations concentration-débit pour les orthophosphates (concentrations en ordonnée et débit en abscisse sont exprimés en valeur relative)
Fig. 5. Relations concentration-débit pour le phosphore total dissous (concentrations en ordonnée et débits en abscisse sont exprimés en valeur relative).
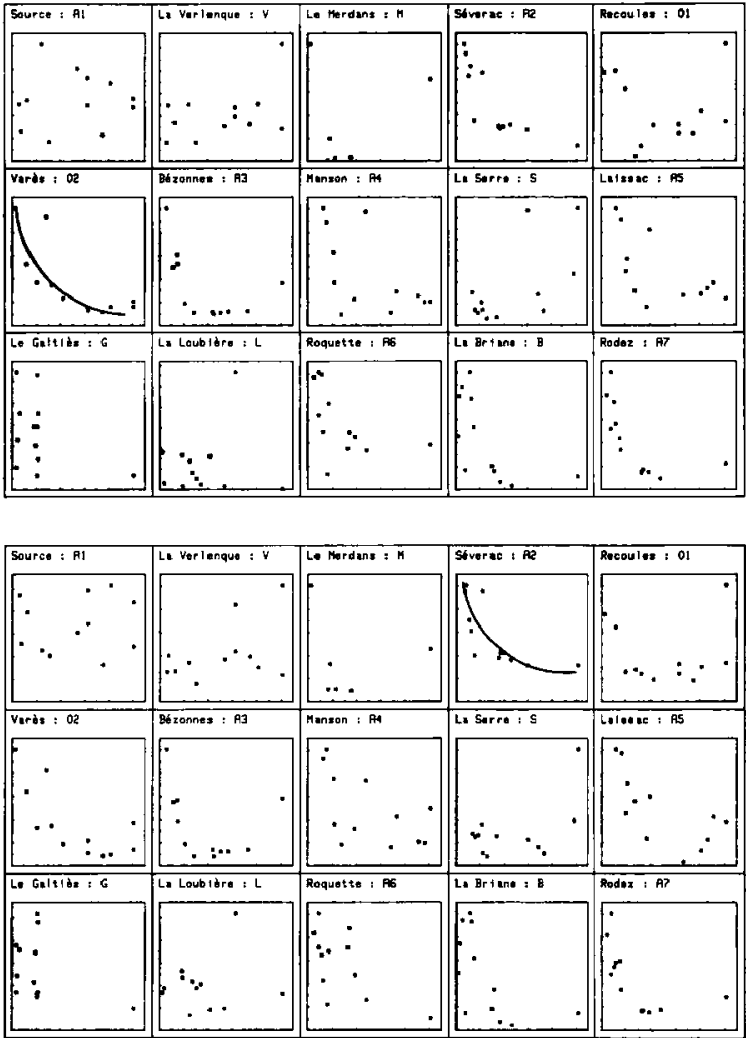

- les rejets ponctuels jouent un rôle important pour les stations A6, A5, A4 et B.

Rejets diffus :

L'examen visuel des relations concentration-débit pour $\mathrm{NO}_{3}$ montre que l'apport par lessivage est marqué pour les stations $\mathrm{V}, \mathrm{O} 1, \mathrm{~A} 4, \mathrm{~L}$ et surtout $\mathrm{B}$, et que ce mécanisme joue un rôle assez important pour les stations $02, \mathrm{~A} 3$ et $\mathrm{A} 6$.

\section{Cas complexe :}

La Serre, à la station $S$, est un exemple de rivière où la fonction $\mathrm{C}=\mathrm{f}(\mathrm{Q})$ est composite. L'examen visuel de la relation $\left(\mathrm{NO}_{3}\right)-\mathrm{Q}$ montre une grande dispersion des points, de même que la relation (ortho$\left.\mathrm{PO}_{4}\right)-\mathrm{Q}$. Il est donc impossible de distinguer un effet de difution (rejet ponctuel) d'un effet de lessivage (rejet diffus).

A l'issue de cette deuxième analyse, on peut tracer une carte indiquant l'origine des apports (fig. 6). 


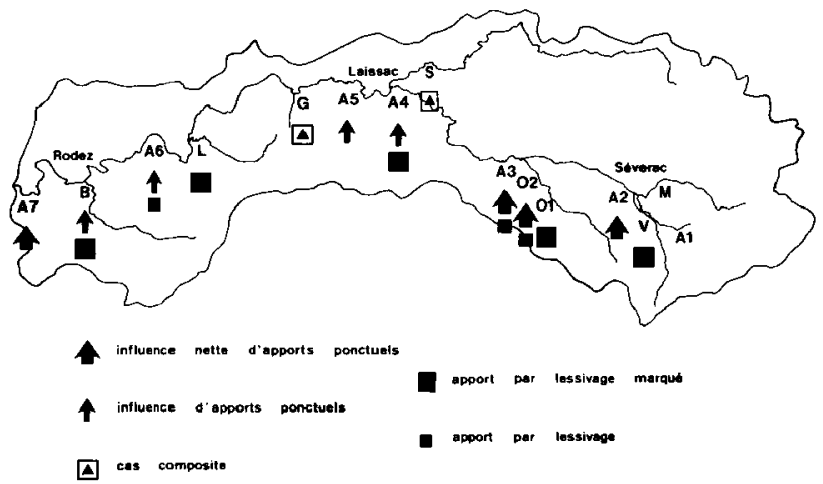

Fig. 6. Représentation schématique des types d'apports.

c) Calcul des flux mensuels.

Les flux globaux (kg. $\left.\mathrm{s}^{-1}\right)$ parvenant à chaque station et dans chaque tronçon ont été calculés pour $\mathrm{NH}_{4}, \mathrm{NO}_{3}$, ortho- $\mathrm{PO}_{4}$ et $\mathbf{P}$ total dissous, chaque mois et dans l'hypothèse d'un régime permanent (tableau II).

Pour chaque station, on calcule donc les quantités :

$\Phi_{\mathrm{x}}^{\alpha} \quad=\quad \mathrm{Q}_{\mathrm{x}} \times \mathrm{C}_{\mathrm{x}}^{\alpha}$

avec : $\Phi_{\mathrm{x}}^{\alpha}=$ flux en constituant $\alpha$ à la station $\mathrm{x}$;

$\mathrm{Q}_{\mathrm{x}}=$ débit à la station $\mathrm{x}$;

$c_{x}^{\alpha}=$ concentration en constituant $\alpha$ à la station $x$

Pour chaque tronçon, on calcule la quantité $R$, différence entre deux flux consécutifs (si R est positif, il y a apport; si $R$ est négatif, il y a perte).

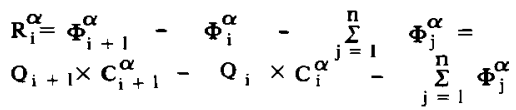

Les stations $i$ (amont) et $i+l$ (aval) sont situées sur l'Aveyron, les $\mathrm{n}$ stations $\mathrm{j}$ sont situées sur les affluents entre $i$ et $i+1$.

Ces calculs permettent l'étude de l'évolution spatiale et saisonnière de ces flux résiduels et leur comparaison avec les flux arrivant aux stations. D'une manière générale, on constate une opposition entre les six premiers et les six derniers mois de l'étude : les flux sont nettement plus faibles au printemps et en été, particulièrement ceux de nitrates, élément pour lequel les concentrations fortes sont associées aux débits élevés (hiver).

- R1 et R6 sont positifs pour les quatre éléments considérés et fortement corrélés, respectivement avec $\mathrm{A} 2$ et avec $\mathrm{A} 7$ :

- R2 est presque toujours négatif pour les quatre éléments ;

- $\mathrm{R} 3$ est positif pour $\mathrm{NH}_{4}$ et $\mathrm{NO}_{3}$; pour le phosphore, R3 est positif de novembre à mars et négatif ou très faible d'avril à septembre ;

- R4 est positif pour ortho- $\mathrm{PO}_{4}$ et $\mathrm{P}$ total dissous ; pour $\mathrm{NO}_{3}, \mathrm{R} 4$ est négatif au printemps et en été et positif en automne et en hiver :

- R5 est positif pour $\mathrm{NO}_{3}$, négatif pour les autres éléments.

Ceci confirme le caractère ponctuel des apports de nutrients au niveau des stations A2 (Sèverac), A5 (Laissac) et A7 (Rodez). Par ailleurs, un phénomène de " perte " de nutrients est mis en évidence sur certains tronçons : en particulier, les flux d'azote et de phosphore se révèlent négatifs tout au long de l'année sur R2, sur R5 (ammoniaque, phosphore), et au printemps et en été sur R3 (phosphore) et R4 (nitrates). 
Tableau II. Flux de nutrient correspondant à chaque station et à chaque tronçon.

1: Ammoniaque (x $0.1 \mathrm{mg} \cdot \mathrm{s}^{-1} \mathbf{N}$ ) ;

2: Nitrates (mg.s.1 N);

3: Orthophosphates (x 0,1 mg.s $\mathbf{~}^{-1} \mathrm{P}$ ) ;

4: Phosphore total dissous ( $0,1 \mathrm{mg} \cdot \mathrm{s}^{-1} \mathrm{P}$ ).

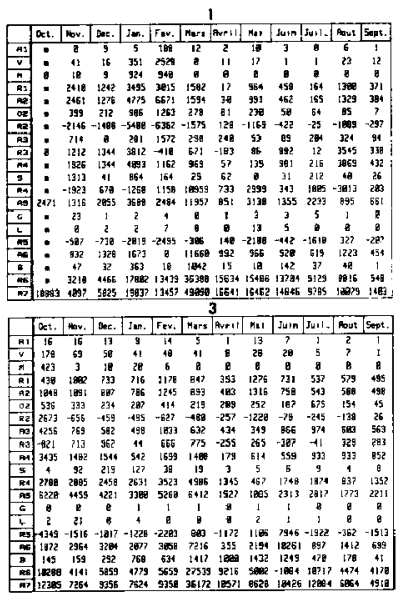

Ces pertes peuvent provenir soit d'une autoépuration par assimilation végétale (macrophytes et périphyton) soit, en ce qui concerne l'azote, d'un phénomène de dénitrification bactérienne. L'utilisation de l'azote par les plantes supérieures dans les eaux n'explique généralement qu'un très faible pourcentage des pertes (Casey 1977). Un calcul simple montre que dans l'hypothèse d'une densité de périphyton correspondant au maximum rencontré dans l'Aveyron $\left(80 \mathrm{~g} . \mathrm{m}^{-2}\right.$ de matières sèches pour $14 \mathrm{~g} . \mathrm{m}^{-2}$ de matière organique), la croissance de ces algues ne peut expliquer qu'au maximum $1 \%$ des pertes de nitrates. En fait, de nombreux auteurs s'accordent à reconnaître l'importance majeure des phénomènes de dénitrification au niveau des sédiments, par réduction bactérienne des nitrates en azote gazeux $\mathrm{N}_{2}$ et oxyde nitreux $\mathrm{N}_{2} \mathrm{O}$ (Kaushik et al. 1981). Ce phénomène estival et printanier enregistré sur de nombreux cours d'eau comparables au Haut-Aveyron peut conduire à des pertes annuelles de plusieurs centaines de kg d'azote sur certains tronçons (Robin-

\begin{tabular}{|c|c|c|c|c|c|c|c|c|c|c|c|}
\hline 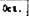 & now. & tore. & $\mathrm{Im}$. & fers. & Marr & Amril & Thar & zoin. & $A+1+1$ & Anour & $\operatorname{sep} x$ \\
\hline 756 & 283 & 218 & 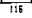 & 139 & 284 & 150 & $2 \overline{256}$ & $12\}$ & 4 & 74 & 35 \\
\hline 19? & 684 & 465 & $4 a z$ & 612 & 507 & 159 & 355 & 19 & $x$ & & \\
\hline & 223 & & 114 & & $n$ & & & & & $\Delta$ & \\
\hline & 551 & 509 & SAB & 93 & 731 & 143 & 156 & !53 & 6s & 3 & 172 \\
\hline & 10 & 1260 & 1380 & 2338 & 1653 & 64 & 336 & 3996 & 64 & 920 & 4 \\
\hline & 8S4 & 6I & 44! & IJE: & i55 & 269 & 334 & 201 & +20 & 140 & 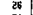 \\
\hline 189 & 112 & If & -4 & 99 & -243 & -35 & & -100 & $-19\}$ & & $-z !$ \\
\hline & 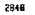 & :423 & 2137 & $29+3$ & $? 17 E$ & 67? & Hag & 舫 & A? & 67 & Eds \\
\hline & 1521 & 3163 & 15! & 3165 & $+4 !$ & $22 ?$ & 859 & r & 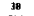 & I $8 \Rightarrow$ & 65 \\
\hline 1993 & 745,5 & โ月5? & AABS & G: & 6534 & 4 & 1?B9 & $?$ & 14 & ?113 & 1 \\
\hline & 2172 & {$[\alpha\}$} & ' 7 & GSE & 5 月. 3 & $32:$ & 5 & $32 R$ & E? & 349 & 2110 \\
\hline & (69\% & 2180 & 65月 & |24 & $-2 \leq 2$ & $-\leqslant \mathrm{H} \overline{3}$ & -gikit & $-21 ?$ & -141 & -9 & \\
\hline & 16285 & & & $?$ & ช9.13 & 837? & $: 273$ & 7๐ & 542 & $2 \times 3$ & 78 \\
\hline & 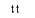 & 11 & 16 & 13 & 56 & 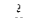 & (d) & ld & 11 & & \\
\hline 116 & 26 & 195 & 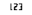 & 9 & टै15 & 36 & 1 & & परे & & \\
\hline & $5:$ & 1 & 1 & 1915 & 14468 & 9 frish & 150 & se & 85 & זי: & \\
\hline & 16754 & 366 & & & $\vec{c} 12 \bar{s}$ & $13 n$ & & [Bi] & 4 & & \\
\hline 唍 & 2477 & :0.2 & 1309 & 1218 & ?s & $=471$ & 392 & 3 & IA? & & 11 \\
\hline 114 & Sat & 3958 & 276 & $0 ? 66$ & 994 & 726 & 43 & latei & 24 & 155 & is \\
\hline & & $1546 \mathrm{~J}$ & 13753 & $15 \mathrm{~T}^{28}$ & sege & $d r$ & & $354 i$ & fte & antion & \\
\hline \\
\hline$x \in$. & $\omega$ & Dee. & I Fn. & rev. & mes & Ansti & MII & \begin{tabular}{|l|} 
Juin \\
\end{tabular} & Jus!. & Areut & Sapt \\
\hline 15 & 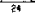 & 12 & 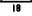 & & & & & - & & & \\
\hline & & & & & & & & & & & \\
\hline 99 & 21 & 18 & & & 17 & & & & & & \\
\hline & 1312 & 59 & & $15 \mathbf{k} 3$ & 1355 & 15 & :Bक⿻ & 615 & i2 & & 38 \\
\hline & 1451 & $11 \%$ & & & 155 & & 7 & & i & & 35 \\
\hline & 25 & $45 e$ & & & 260 & & & & Dis & & \\
\hline & -8 & -873 & -11 & $-E x$ & -893 & -116 & $-\{B\}$, & -156 & -424 & $-13 x$ & \\
\hline & & & & & $m$ & & & & 1800 & & \\
\hline & 32 & 17 & 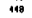 & & 24 & & & & פו & & \\
\hline & 13 & & a & is & 1528 & 398 & 964 & 20 & 1154 & 1216 & 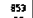 \\
\hline & & & & & 53 & & & & & & \\
\hline & 5268 & -75 & $-A B j$ & 319 ? & 日1:1 & 33 & 99 & & 85 & & \\
\hline & 9654 & 26 & $=7$ & & 7724 & in & et & 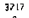 & 3892 & 2259 & 286 \\
\hline 1 & 5 & 5 & 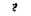 & & 5 & & & & & & \\
\hline B & 4 & 7 & & & 2 & & & & & & \\
\hline & -6750 & 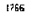 & 58 & 3 & A & - 2999 & & $-258 \mathrm{a}$ & -2592 & - - - & 200 \\
\hline & 396 & 4391 & & & . & & & & 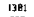 & & \\
\hline & 251 & 34 & 769 & 1563 & 2的的 & 98 & 1500 & 1672 & 565 & 1 & 4 \\
\hline & 13037 & 725 & 4980 & 274 & SAB85 & 15926 & G9a2 & 18623 & 13247 & 5869 & $53 \%$ \\
\hline & 170 & U⿴囗十⺝ & 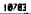 & 11478 & & & 11119 & 13442 & I5IE & 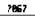 & 518 \\
\hline
\end{tabular}

son et al. 1979) ; ces pertes peuvent représenter $50 \%$ de l'apport en azote et $75 \%$ de l'apport en nitrates (Hill 1979).

En conclusion de cette troisième analyse, on peut tracer une carte montrant les apports essentiels et les secteurs marqués par des phénomènes d'assimilation végétale et essentiellement de dénitrification bactérienne (fig. 7 ).

\section{3. - Conclusion}

La méthode suivie a permis de mettre en évidence plusieurs caractéristiques du réseau hydrographique du Haut-Aveyron. (1) La chimie de l'eau, avec des taux généralement élevés d'azote et de phosphore, favorise le développement de la végétation aquatique ; par ailleurs, les plus fortes concentrations sont atteintes pour les faibles débits, au printemps et en été. (2) Les quantités de nutrients issues des bassins forestiers sont très faibles (cas des 


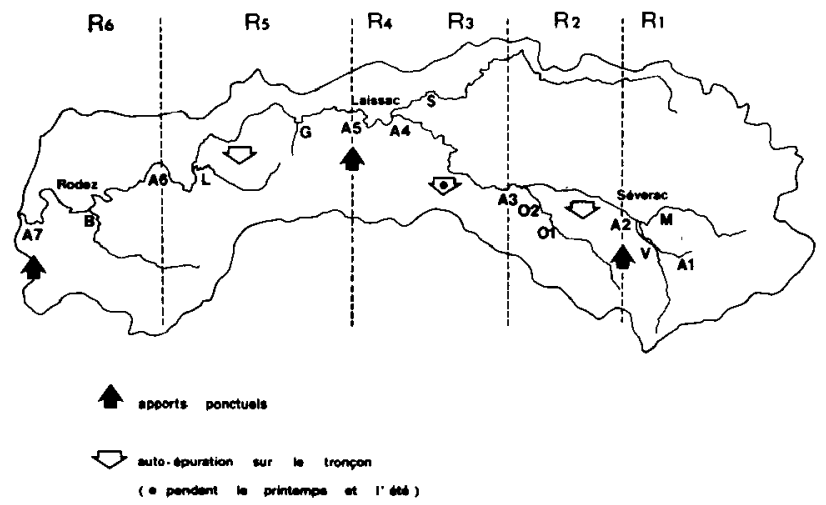

Fig. 7. Localisation des apports essentiels et des phénomènes d'autoépuration.

affluents la Verlenque, le Galtiès, la Loubière). (3) L'effet des zones cultivées sur la charge en nutrients se manifeste sur la Briane ; cet effet se trouve cependant partiellement masqué par l'existence de rejets ponctuels. (4) Certains tronçons de l'Aveyron et de quelques affluents (la Serre) ont un rôle autoépurateur (phénomènes de dénitrification bactérienne). (5) Les trois localités les plus importantes du bassin versant (Sèverac, Laissac et surtout Rodez) sont à l'origine d'importantes pollutions ponctuelles.

\section{Travaux eltes}

Belamie (R.). 1978. - Transfert des fertilisants vers les caux de surface dans un petit bassin versant agricole. Verh. Internat. Verein Limnol, $20: 1503.1508$.
Casey (H.). 1977. - Origin and variation of nitrate-nitrogen in the chalk springs, streams and rivers in Dorset, and its utilisation by higher plants. Prog. Wat. Tech, 8 (4/5) : 225-235.

Chauvet (E.). 1981, - Eutrophisation du Haut-Aveyron. Thèse de 3* cycle. Univ. Toulouse : $97 \mathrm{p}$.

Hall (R.) 1970. - Dissolved solids discharge relationships 1 mixing models - Wat. Res. Research, 6 (3) : 845-850.

Hill (A.R.). 1979. - Denitrification in the nitrogen budget of a river ecosystem. Nature, $281: 291-292$.

Kaushik (N.K.), Robinson (J.B.), Stammers (W.N.) \& Whiteley (H.R.). 1981. - Aspects of nitrogen transport and transformation in headwater streams. In : Perspectives in Running Water Ecology, Lock M.A. \& Williams D.D. (eds). Plenum Press, New York: 113.139.

Manczak (H.) \& Florczyck (H.). 1971. - Interpretation of results from studies of pollution of surface flowing water. Water Research, 5 (8) : 575-584

Robinson (J.B.), Whiteley (H.R.), Stammers (W.N.), Kaushik (N.K.) \& Sain (P.). 1979. - The fate of nitrate in small streams and its management implications. In : Best managemert practices for agriculture and silviculture. Proceedings of the 10th Annual Comell Agricultural Waste Management Conference. R.C. Loehr, D.A. Haith. M.F. Walter and C.S. Martin (eds), 1978. Ann Arbor Sci. Publishers Inc., Ann Arbor, M.I.
Cette étude a été réalisée dans le cadre d'un programme P.I.R.E.N. Finance par l'Agence Financiere de Bassin AdourGaronne (convention $n^{\circ} \mathrm{E} / 09 / 79$ ). 